\title{
A análise gödeliana do conceito de percepção*
}

Sérgio Schultz

sergioschultz@yahoo.com.br

Pontifícia Universidade Católica do Rio de Janeiro, Rio de Janeiro, RJ, Brasil

resumo No presente artigo expomos alguns aspectos das concepções gödelianas acerca da percepção. Procuramos mostrar aqui que a comparação realizada por Gödel entre percepção sensivel e racional, bem como suas concepções sobre esta última, correspondem a uma análise do conceito de percepção. Em uma primeira seção, expomos a analogia gödeliana entre percepção sensível e racional, salientando que ambas são comparadas tendo em vista seu status de relação de acesso a entes. A partir disto, argumentamos, na segunda seção, que subjazendo à analogia há uma análise do conceito de percepção como o conceito geral ou formal de uma relação de acesso, do qual a percepção sensível e a racional são casos particulares. Tal análise, como defendemos, acaba por ser mais adequada ao uso comum que fazemos deste conceito do que pareceria à primeira vista.

palavras-chave Percepção; Percepção sensível; Percepção racional; Gödel; Acesso; Platonismo

Em vários lugares na obra de Gödel, encontramos a defesa de uma percepção racional, e um elemento fundamental de tal defesa é a analogia entre as percepções sensível e racional - ou percepção de conceitos. A analogia de Gödel costuma ser entendida como mostrando que, bem como não haveria porque recusar a percepção sensível, também não haveria porque recusar a percepção de conceitos. De fato, a analogia cumpre com esta função. Contudo, subjazendo a analogia e a defesa da percepção racional, há também uma análise do conceito de percepção. É esta análise do que investigamos neste artigo.

Recebido em 20 de maio de 2012. Aceito em 13 de setembro de 2012.

doispontos, Curitiba, São Carlos, vol. 9, n. 2, p.135-150, outubro, 2012 
Em um primeiro momento, expomos a analogia entre percepção racional e sensível, salientando que ambas são comparadas tendo em vista seu status como uma relação de acesso a entes. A partir disto, argumentamos, na segunda seção, que subjazendo a analogia há uma análise do conceito de percepção como o conceito geral ou formal de uma relação de acesso, do qual a percepção sensível e a racional seriam casos particulares. Tal análise, como defendemos, acaba por ser mais adequada ao uso comum que fazemos deste conceito do que pareceria à primeira vista.

\section{A analogia entre percepção sensivel e percepção intelectual}

Em seu artigo sobre a hipótese do contínuo, Gödel afirma que nós possuímos algo como uma percepção dos objetos da teoria de conjuntos, apesar destes não serem objetos da percepção sensível, e que esta percepção seria responsável pelo do fato dos axiomas da teoria de conjuntos se forçarem sobre nós como verdadeiros. Ambas, percepção sensível e percepção racional, seriam análogas. A diferença entre a percepção dos objetos da teoria de conjuntos - percepção racional ou percepção de conceitos - e a percepção sensível é que os dados da primeira não seriam sensações e, como opostos a sensações, sua presença em nós resultaria de um tipo diferente de relação com a realidade ${ }^{1}$. Embora esta não seja nem a primeira nem a única ocorrência na obra de Gödel das ideias de percepção intelectual e da analogia entre esta e a percepção sensível, ela é, sem dúvida, a mais citada. Além desta passagem, encontramos afirmações sobre intuição intelectual e sobre a analogia entre esta e a percepção sensível também no livro de Wang (1974), na

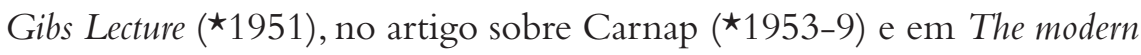
development of the foundations of mathematics in the light of philosophy ( $\star 1961 /$ ?).

Aparentemente, Gödel estaria defendendo apenas que, em primeiro lugar, nós temos intuição matemática (também referida como "percepção/intuição intelectual" ou "percepção/intuição de conceitos") e, em segundo lugar, que esta é tão confiável quanto a percepção sensível. Porém, um exame mais atento da analogia entre percepção sensível e intuição de conceitos, bem como da natureza desta última, revelam que, 
subjazendo a estas afirmações, encontra-se uma análise do conceito de percepção. Isto se torna mais claro ao lembrarmos o problema que o apelo à intuição intelectual pretende resolver e o papel que a analogia desempenha nesta proposta de solução.

Ainda que seja claro que Gödel não esteja debatendo com Benacerraf, o problema que ele está abordando quando propõe sua noção de percepção racional é o problema do acesso aos entes conhecidos, levantado por aquele autor no artigo Mathematical Truth. O conhecimento matemático se dá, em parte, por meio de provas. Contudo, toda prova deve partir de verdades que não podem mais elas mesmas ser demonstradas, os axiomas. Assim, enquanto que podemos recorrer à noção de prova para responder à pergunta sobre como conhecemos a verdade dos teoremas, o mesmo não pode ser feito, de um ponto de vista platonista, com respeito aos axiomas. Portanto, nosso conhecimento dos axiomas demanda alguma relação entre nós e os objetos ou conceitos conhecidos ou, então, entre nós e os fatos que os tornam verdadeiros, e tal relação seria a de percepção racional.

Na obra de Gödel, é verdade, problema do acesso adquire uma qualificação. No artigo sobre a Hipótese do Contínuo, bem como em outros textos nos quais Gödel aborda o problema da percepção racional, ele não está concernido - ao menos não primariamente - com o conhecimento de axiomas já estabelecidos. Antes, o problema do acesso em Gödel é formulado tendo como pano de fundo seus resultados de incompletude. Desta forma, o problema não se coloca em termos de como conhecemos os axiomas da teoria, mas sim em termos de como podemos decidir enunciados que são demonstráveis a partir dos axiomas disponíveis como, por exemplo, é o caso da Hipótese do Contínuo no contexto da teoria de Zermelo-Fraenkel.

O aspecto ao qual nos referimos acima, porém, é apenas uma nuance particular que o problema adquire para Gödel, em parte porque ele não pensa o problema apenas de uma perspectiva filosófica, mas também da perspectiva de um praticante das teorias em questão. Para alguém envolvido, digamos, em tentativas de provar ou refutar a Hipótese do Contínuo, a questão que assume primazia é sobre a justificação de novos princípios a partir dos quais a hipótese poderia ser decidida, e não a pergunta sobre a justificação de princípios já aceitos e que não nos 


\section{8}

permitem decidir a questão. $\mathrm{O}$ cerne da questão, no entanto, segue sendo o mesmo: como conhecemos a verdade de enunciados matemáticos que não podem mais, por uma razão ou outra, ser demonstrados.

O mesmo problema descrito acima para o conhecimento matemático também se coloca no caso empírico: algumas verdades empíricas podem ser conhecidas por meio de inferências. Porém, tais inferências pressupõem premissas verdadeiras que, em última instância, não podem mais ser conhecidas inferencialmente. O conhecimento destas premissas, então, demanda uma certa relação entre nós e os objetos ou fatos conhecidos. Tanto no caso empírico quanto no caso matemático, o conhecimento demanda algum tipo de relação com os objetos ou conceitos conhecidos.

No caso empírico, a relação necessária para o conhecimento poderia ser pensada como sendo a percepção sensível. O que Gödel defende é que, no caso matemático, a relação em questão é a de percepção racional (ou percepção intelectual ou, ainda, intuição matemática). Além disto, ele também defende que esta relação de percepção racional seria análoga à percepção sensível. O problema, agora, é entender como a analogia entre percepção sensível e racional intelectual se neste quadro, i. e., qual é a função que ela desempenha dentro da argumentação gödeliana. Para tanto, é necessário entender melhor quais são os termos nos quais a analogia é formulada e qual é sua natureza.

A percepção sensível e a percepção intelectual são comparadas por Gödel tendo em vista alguns aspectos que são essenciais para que uma relação com entes se constitua em uma relação de acesso. Um destes aspectos, e que é o aspecto mais saliente da analogia, é o que poderíamos chamar do caráter não-subjetivo da relação de acesso.

Tanto percepção intelectual quanto percepção sensível seriam, em grande medida, independentes de nossas crenças ou de nossa vontade. Não é porque queremos acreditar ou acreditamos que o objeto seja vermelho que o percebemos como sendo vermelho, mas sim é porque o objeto é vermelho que nós o percebemos como tal. Não cabe a nós decidir como percebemos o objeto. Outra forma de dizer isto consiste em afirmar que não é nossa crença na verdade de "este objeto é vermelho" - ou nosso desejo de que aquele enunciado seja verdadeiro - que nos faz perceber o objeto como sendo vermelho. Antes, é a percepção do objeto vermelho que força sobre nós aquele enunciado como sendo 
verdadeiro. Ao afirmar que a percepção dos objetos da teoria de conjuntos força sobre nós como verdadeiros os axiomas, Gödel está salientando o caráter não-subjetivo da percepção de conceitos

Outro aspecto segundo o qual percepção sensível e racional seriam análogas diz respeito aos limites e à falibilidade da percepção. Bem como a percepção sensível pode ser indistinta e enganosa, como no caso do bastão parcialmente imerso na água, também a percepção de conceitos pode ser indistinta e enganosa, como seria o caso, por exemplo, com os paradoxos da teoria de conjuntos ${ }^{2}$. Algo que, de início, parecia ser um único conceito pode acabar se mostrando como sendo, na verdade, dois conceitos distintos.

O mesmo pode ocorrer com a percepção de objetos concretos ${ }^{3}$. Inclusive, o modo como descobrimos os enganos ou ilusões seriam similares em ambos os casos, a saber, alcançando uma inconsistência. No caso da percepção de entes abstratos, as ilusões mostram-se através da derivação de uma contradição, e os paradoxos da teoria de conjuntos são mencionados por Gödel nesse contexto. No caso da percepção sensível, as ilusões vêm à tona através de uma inconsistência entre diferentes observações. Como um exemplo, podemos mencionar que a percepção visual de que o bastão semimergulhado está quebrado pode ser concebida como uma ilusão na medida em que contradiz nossa percepção táctil do mesmo bastão. ${ }^{4}$

Um aspecto adicional da analogia que está implícito aqui é a possibilidade de descobrirmos a ilusão e de explicar porque ela ocorre. No caso empírico, a ilusão ocorre devido às diferenças no modo como a luz é refletida pelo bastão através da água e através do ar, pela parte do bastão fora da água. Nos casos daquilo que seriam ilusões da percepção de conceitos, algumas delas se dariam uma vez que confundimos dois conceitos pensando se tratar de um só, e a explicação de por que confundimos os conceitos é também a explicação de por que tal ilusão ocorreu. Poderíamos, assim, identificar e explicar aquelas circunstâncias nas quais a percepção de conceitos mostra-se enganosa. Além disso, podemos também ter percepções cada vez mais claras de conceitos, bem como ocorre com a percepção sensível de objetos concretos. ${ }^{5}$

Também, haveria uma quantia ilimitada de percepções, tanto da percepção de objetos sensíveis quanto da percepção de entes abstratos, o 
quê, neste último caso, seria exemplificado pela série ilimitada de axiomas de infinito em teoria de conjuntos ${ }^{6}$. A esse caráter ilimitado da percepção, seja ela sensível ou intelectual, soma-se a possibilidade de percebermos, tanto objetos físicos quanto conceitos, de diferentes perspectivas. Com respeito a objetos físicos, podemos simplesmente nos mover ao redor do objeto e, assim, enxergá-lo sob diversos ângulos. O análogo disso na percepção de conceitos se daria, segundo Gödel, através de diferentes conceitos logicamente equivalentes ${ }^{7}$.

O que está em jogo na analogia é a possibilidade e os meios disponíveis para conhecermos entes abstratos e, nesse contexto, Gödel argumenta que mantemos uma relação com conceitos que possui todas as características necessárias para que possamos, com base nessa relação, fundamentar enunciados matemáticos. Em outras palavras, nós possuímos com entes abstratos uma relação que é não-subjetiva, que é falível porém corrigível e que é tal que podemos explicar aqueles casos nos quais esta relação nos forneceria 'resultados' errados - os casos nos quais ela nos 'enganaria'8. A analogia entre percepção sensível e percepção de conceitos é realizada exatamente em termos destas características.

Um último aspecto da analogia de Gödel que devemos salientar diz respeito ao papel desempenhado pela percepção de conceitos e pela percepção sensível. Na medida em que a percepção de conceitos (ou percepção racional) possui as características mencionadas acima, ela nos permitiria confirmar nossas teorias acerca daqueles domínios. Isto se daria seja de forma direta, por exemplo, com a percepção dos objetos conjuntistas forçando sobre nós como verdadeiros os axiomas da teoria, seja de forma indireta, através das consequências que os axiomas possuem a respeito de domínios onde temos intuições mais claras 9 . De modo análogo, a percepção sensível nos permitiria confirmar teorias acerca de objetos concretos, seja por observação direta, seja verificando, por meio da percepção, as consequências das leis acerca daqueles objetos.

\section{0 Conceito de percepção}

Como se nota a partir da descrição acima, a analogia entre percepção sensível e percepção racional é formal. Ela se estabelece, por um lado, em 
virtude do papel que percepção sensível e percepção/intuição intelectual desempenhariam, respectivamente, no conhecimento de objetos concretos e de entidades abstratas. Por outro lado, a analogia também envolve certas características comuns à percepção sensível e à percepção racional que possibilitam que esta última assuma, com respeito ao conhecimento de entes abstratos, o mesmo papel que a percepção sensível assume com respeito a entes concretos. ${ }^{10}$

Enquanto que formalmente análogas, os dois tipos de percepção se distinguiriam pelo seu conteúdo. A percepção sensível é uma relação que envolve necessariamente contato causal com aquilo que é percebido, o mesmo não se passa com a percepção racional, cujos objetos são abstratos e, portanto, causalmente inertes. Desta forma, como afirma Gödel, os dados da percepção sensível e se distinguiriam dos dados da percepção de conceitos por resultarem de diferentes relações com a realidade. Os dados da percepção sensível resultariam da relação causal entre os objetos percebidos e nosso aparato perceptual (entendido como constituído por nossos órgãos dos sentidos): nós percebemos sensivelmente as coisas através de nossos cinco sentidos. Nós descrevemos tal relação dizendo "eu vi (com meus olhos)...", “eu ouvi...", “eu tateei...”, etc.

Esta outra relação com entes, que nos forneceria os dados da percepção racional, se daria entre entes abstratos (conceitos) e nossa razão, e nós a descreveríamos dizendo "eu compreendo...". Tais aspectos são salientados por Gödel em várias passagens tanto de seus escritos não publicados quanto de suas conversas com Wang. Deste modo, na versão IV de seu artigo dedicado a Carnap, Gödel resume as relações entre sensibilidade e objetos particulares e razão e conceitos do seguinte modo:

Pois enquanto que com o ultimo [os sentidos] nós percebemos coisas particulares, com a razão nós percebemos conceitos (acima de tudo, conceitos primitivos) e suas relações. ((MS, p. 17), como citada por Parsons em (PARSONS, 1995, p. 63)). ${ }^{11} 12$

Já sobre a relação entre percepção racional e compreensão, 7.3.12 Conjuntos são objetos, mas conceitos não são objetos. Nós percebemos objetos e compreendemos conceitos. Compreensão é um tipo diferente de percepção: ela é um passo na direção da redução à última causa (WANG, 1996, p. 235). ${ }^{13}$ 
7.3.4 Tentar ver (.i.e. compreender) um conceito mais claramente é o modo correto de descrever o fenômeno descrito vagamente como “examinar o que uma palavra quer dizer" (WANG, 1996, p. 233, ênfase minha; esta última afirmação é relatada também em (WANG, 1974, p. $85))^{14}$.

Considerando esta forma de entender a noção gödeliana de percepção racional, começa a vir à tona uma análise do conceito de percepção que se distingue das análises usuais na medida em que leva em consideração os usos que fazemos do conceito de percepção com referência a entes abstratos.

O que merece ser ressaltado, aqui, é que Gödel não está postulando uma misteriosa faculdade de percepção extrassensorial. $\mathrm{Na}$ verdade, embora Gödel não tivesse simpatia pela filosofia da linguagem comum, suas teses acerca da percepção são bastante fiéis ao uso ordinário do verbo "perceber" em dois aspectos. Primeiro, que tal verbo possui um uso muito mais amplo do que aquele no qual falamos que um objeto concreto é percebido pelos sentidos. Comumente, nós dizemos coisas como:

(1) "X percebe tal-e-tal objeto à sua frente"

(2) "X percebe as relações entre tais-e-tais conceitos"

(3) "X percebe do que se trata tal-e-tal situação"

(4) "X percebe que tal-e-tal é o caso"

(5) "X percebe o que $Y$ quer dizer/o que significa tal-e-tal coisa"

(6) "X percebe como tal-e-tal coisa funciona"

(7) " $X$ percebe $Y$ como um $Z$ "

Etc.

Destes, apenas (1) representa um caso no qual a percepção pode ser entendida como percepção sensível. Em todos os demais, aquilo que é percebido é em boa medida abstrato: relações entre conceitos, fatos ou verdades, modos de funcionamento, significados, etc. Afirmar que nem toda percepção é sensível ou que existem formas não sensíveis de percepção remonta apenas a reconhecer que, da mesma forma que usamos o conceito de percepção como em (1), nós também o usamos como em (2)-(7). Se, além disso, assumimos uma posição platonista, de acordo com a qual conceitos, verdades e significados são entidades platônicas, pelo menos em (2), (4) e (5) nós temos enunciados significa- 
tivos que dizem que alguém $(X)$ possui percepção de uma entidade abstrata e causalmente inerte.

O segundo aspecto no qual Gödel está sendo fiel ao uso comum do verbo "perceber" diz respeito a que, enquanto que o primeiro caso é um no qual perceber é ouvir, enxergar, tatear, etc., nos demais casos a percepção em questão é um compreender, i. e., é percepção racional:

(2a) "X compreende as relações entre tais-e-tais conceitos"

(3a) "X compreende do que se trata tal-e-tal situação"

(4a) " $X$ compreende que tal-e-tal é o caso"

(5a) " $X$ compreende o que $Y$ quer dizer/o que significa tal-e-tal coisa"

(6a) " $X$ compreende como tal-e-tal coisa funciona"

(7a) " $X$ compreende $Y$ como um $Z$ "

Compreender, por sua vez, é uma atividade eminentemente racional: não tem sentido falar de compreensão sensível ou de compreender com os sentidos. Nós compreendemos através da razão e, portanto, aquela percepção que remonta a um compreender é percepção racional.

Poder-se-ia argumentar que em (2) - (7) o verbo "perceber" não está sendo usado em seu sentido literal, mas sim como uma metáfora para compreensão. O mesmo ocorre quando dizemos "vemos que tai-e-tais conceitos se relacionam assim-e-assim”, onde é claro que não estamos querendo dizer que enxergamos as relações entre os conceitos e que o verbo "ver" é usado metaforicamente. No entanto, é útil considerar aqui como poderíamos justificar a afirmação de que toda percepção é percepção sensível, isto é, de que o conceito de percepção é tal que não possui sentido literal falar de percepção de entes abstratos ou de percepção racional.

Para defender que expressões como "perceber uma verdade" e "perceber um conceito" são metafóricas é necessário, no mínimo, mostrar que a palavra "perceber", nestes contextos, não possui de modo algum o mesmo sentido que possui em contextos nos quais pode ser entendida como percepção sensível.Vale lembrar que o que está em questão é justamente se toda percepção é sensível ou não, e que esta questão é a contraparte da pergunta sobre se os usos abstratos do verbo "perceber" são metafóricos ou se eles possuem sentido literal. Tais usos possuem sentido literal se e somente se a percepção não se restringe à percepção sensível; 


\section{4}

e a percepção não se restringe à percepção sensível se e somente se tais usos possuem sentido literal. Sendo assim, não poderíamos afirmar que expressões como "perceber que é verdade" ou "perceber um conceito" somente possuem sentido se entendidas metaforicamente porque toda percepção é sensível, e nem poderíamos defender o oposto: isto seria uma petição de princípio.

Nós, de fato, usamos o verbo "perceber" com referência a entes abstratos. Para justificar a afirmação de que tais usos são metafóricos - e que toda percepção é sensível - é necessário mostrar que não há um único conceito de percepção operando tanto em contextos concretos quanto em contextos abstratos. Para mostrar que os usos abstratos do verbo "perceber" não são metafóricos, é necessário mostrar que há um mesmo conceito de percepção operando nos usos abstratos e nos concretos. O que está em jogo é uma análise do conceito de percepção e, a nosso ver, um dos pontos da analogia entre percepção racional e sensível consiste justamente em mostrar que existe um tal conceito de percepção, do qual a percepção racional e a sensível seriam casos particulares. Em outras palavras, ao comparar percepção sensível e racional nos termos descritos na seção anterior - caráter objetivo ou não-subjetivo, corrigibilidade, possibilidade de explicar os enganos de percepção - Gödel não está procurando mostrar somente que a percepção racional é tão confiável quanto a percepção sensível. Ele também está exibindo aquele conceito comum de percepção que nos permite tomar os usos abstratos do verbo "perceber" - (2)-(7) acima - literalmente.

Isto se torna mais claro quando consideramos o modo como Gödel introduz a noção de percepção racional no artigo sobre o problema do Contínuo. Ali, ele afirma:

Mas, a despeito de seu afastamento da percepção sensível, nós possuímos algo como uma percepção também dos objetos da teoria de conjuntos, como se vê pelo fato de que os axiomas se forçam sobre nós como sendo verdadeiros $\left(1964\right.$, p. 268). ${ }^{15}$

Embora no restante do parágrafo que contém esta passagem a atenção de Gödel se volte para a questão da confiabilidade da percepção racional ou de conceitos, nesta passagem em particular ele está argumentando pela existência deste tipo de percepção, e ela se seguiria do fato de que os 
axiomas se forçam sobre nós como verdadeiros. Que os axiomas se forçam sobre nós como verdadeiros implica que possuímos acesso aos objetos (os conceitos) dos quais tratam os axiomas: mantemos com tais objetos uma relação que nos dá a saber a verdade dos axiomas. Do fato de termos acesso se seguiria, por sua vez, que possuímos percepção dos entes em questão.

Pareceria, então, que faz parte do conceito de percepção racional que ele diz respeito a uma relação de acesso a entes. É uma condição suficiente para uma determinada relação com entes ser a relação de percepção que ela seja uma relação de acesso. Desta forma, do fato de termos acesso aos objetos (ou conceitos) da teoria de conjuntos, se segue que, segundo Gödel, que possuímos percepção, no caso, racional, deles.

A diferença entre percepção racional (ou de conceitos) e percepção sensível seria que, na primeira, o acesso se daria através da razão, na segunda, ele se daria através dos sentidos. O que é relevante aqui, porém, é que o fato de ambas serem uma relação de acesso nos permitiria considerar percepção racional quanto percepção sensível como casos do conceito de percepção. Seguindo esta linha de interpretação, o conceito de percepção seria analisado como sendo o conceito geral - ou, talvez, formal - de uma relação de acesso, i. e., uma relação com entes nos permite saber algo acerca dos entes com os quais mantemos tal relação. Isto não se encontra explicito em Gödel, mas nos parece ser o que subjaz sua analogia e a ideia de que tanto percepção sensível quanto percepção racional são tipos de percepção. Também, o fato da analogia ser colocada nos termos daquilo que faz com que uma relação com entes seja uma relação de acesso, como defendemos na seção anterior, reforça esta ideia.

Talvez seja precipitado concluir que Gödel defende que percepção e acesso são um e o mesmo conceito ou que sua posição implicava tal identificação. Contudo, no mínimo isto pareceria implicar que uma característica central da relação de percepção $-x$ percebe $y-$ é que ela seja uma relação de acesso. Também, refletindo sobre os usos (1) - (7) elencados acima, parece ao menos plausível que a relação de percepção seja, de fato, uma relação de acesso.

Desta forma, em todos eles pareceria ser parte do significado da sentença que é mantida uma relação com algo (a de $X$ perceber $Y$ ) e que esta relação é tal que ela dá a saber algo acerca do ente (abstrato ou não) 


\section{6}

com o qual mantemos esta relação. Por exemplo, aquele que diz perceber como funciona algo implica, com isto, que sabe como aquilo funciona: se ele alega não saber como funciona o objeto em questão, então podemos concluir que ele não percebeu como ele funciona. Da mesma forma, quem diz ter percebido que algo é verdade implica, com isto, que sabe que aquilo é verdade. Também, com relação ao último dos usos elencados - (7) $X$ percebe $Y$ como um $Z$ - a percepção pareceria dizer respeito justamente ao modo de acesso ao ente $Y$. Por exemplo, quando alguém diz algo como "físicos percebem os objetos como compostos de partículas, biólogos os percebem como composto por células", pareceria que se está falando sobre o modo de acesso a objetos que é peculiar à física e à biologia.

Bem como Gödel concorda com a linguagem comum ao admitir percepção não sensível e ao interpretá-la como sendo compreensão, não seria absurdo supor que também aqui sua análise concorda com o uso comum do verbo "perceber" e entende o conceito de percepção como o conceito geral de uma relação de acesso. Claro, não estamos de modo algum estamos sugerindo que Gödel alguma vez tenha se engajado na análise do uso cotidiano da linguagem. Porém, suas concepções acerca dos conceitos de percepção e de percepção se mostram próximas daquelas que podemos extrair de uma reflexão sobre o uso comum, a ponto de conjecturarmos que era um entendimento do conceito de percepção como uma relação de acesso que subjazia suas teses epistemológicas. Embora a concordância com o uso comum da linguagem não se constitua em um critério de correção para concepções filosóficas, tal concordância no mínimo fala a favor da plausibilidade de uma concepção.

\section{Conclusão}

A famosa analogia de Gödel entre percepção de conceitos e percepção sensível não envolve somente uma tese acerca do conhecimento de entes abstratos. Ela envolve também uma tese acerca do conceito de percepção. Esta tese pode ser formulada dizendo que o conceito de percepção não se restringe ao conceito de percepção sensível. Quando formulada desta forma, é trivial que Gödel a sustentou. Afinal, ele defendia a existência de 
uma forma não-sensível de percepção. O que pretendemos ter salientado neste artigo é que a percepção racional não é simplesmente uma postulação de Gödel. Antes, ela seria parte de uma análise do conceito de percepção que, apesar do que poderia parecer à primeira vista, se mostra muito mais de acordo com o modo como usamos este conceito do que aquelas concepções que restringem o conceito de percepção ao de percepção sensível.

* Este artigo foi escrito como parte do projeto de pós-doutorado financiado pela FAPERJ/CAPES intitulado Intuição Intelectual e Conhecimento Simbólico. Gostaria de agradecer a Frank Thomas Sautter (UFSM), Abel Lassalle Casanave (UFBA), Gisele Dalva Secco (Doutoranda/PUC-Rio), Daniel Simão Nascimento (Doutorando/PUC-Rio) e, em especial, a Oswaldo Chateaubriand (PUC-Rio), por comentários, críticas e sugestões a versões preliminares do presente trabalho.

${ }^{1}$ But, despite their remoteness from sense experience, we do have something like a perception also of the objects of set theory, as is seen from the fact that the axioms force themselves upon us as being true. I don't see any reason why we should have less confidence in this kind of perception, i.e., in mathematical intuition, than in sense perception, which induces us to build up physical theories and to expect that future sense perceptions will agree with them, and, moreover, to believe that a question not decidable now has meaning and may be decided in the future. It should be noted that mathematical intuition need not be conceived of as a faculty giving an immediate knowledge of the objects concerned. Rather it seems that, as in the case of physical experience, we form our ideas also of those objects on the basis of something else which is immediately given. Only this something else here is not, or not primarily, the sensations. [...] they [os dados da percepção intelectual], too, may represent an aspect of objective reality, but, as opposed to the sensations, their presence in us may be due to another kind of relationship between ourselves and reality. (GÖDEL, 1964, p. 268)

${ }^{2}$ Veja (GÖDEL $\star 1951$, p. 34).

${ }^{3}$ Veja (WANG, 1974, pp. 85-86).

${ }^{4}$ Este exemplo, assim como este segundo aspecto da analogia, encontra-se em Gödel ( ${ }^{\star} 1951$, p. 34): our knowledge of the world of concepts may be as limited and incomplete as that of [the] world of things. It is certainly undeniable that this knowledge, in certain cases, not only is incomplete, but even indistinct. This occurs in the paradoxes of set theory, which are frequently alleged as a disproof of Platonism, but, I think, quite unjustly. Our visual perceptions sometimes contradict our tactile perceptions, for example, in the case of a rod immersed in water, but nobody in his right mind will conclude from this fact that the outer world does not exist.

${ }^{5}$ Veja (WANG, 1974, p. 84ss). 
${ }^{6}$ Veja (GÖDEL, *1953/9-III, \$41, n. 43). Este aspecto também se faz presente na ideia, exposta em Wang (1974, p. 324), de que a mente humana é incapaz de formular todas as suas intuições matemáticas.

${ }^{7}$ Veja (WANG, 1974, p. 85).

8 Sobre tais características relacionadas ao conceito de percepção sensível, veja (AZZOUNI, 1997).

${ }^{9}$ Veja (WANG, 1974, p. 265 e 272).

10 Parsons, em (1995, pp. 62-64), também caracteriza a analogia fornecida por Gödel como sendo formal. Contudo, enquanto que Parsons parece criticar Gödel por fornecer apenas uma analogia formal, nós acreditamos que aquilo que é de fato relevante e substancial para a concepção defendida por Gödel é justamente que a analogia seja formal.

${ }^{11}$ For while with the later [the senses] we perceive particular things, with reason we perceive concepts (above all the primitive concepts) and their relations.

${ }^{12}$ Veja também (GÖDEL, ^1953/9-II, \$39, p. 1852 versão III, \$42). Na Gibbs Lecture, logo após expressar sua confiança de que o platonismo é a única perspectiva viável, Gödel caracteriza o platonismo afirmando: thereby [a perspectiva platonista] I mean the view that mathematics describes a non-sensual reality, which exists independently both of the acts and the dispositions of the human mind and is only perceived, and probably perceived very incompletely, by the human mind. (GÖDEL, ^1951, p. 38)

13 7.3.12 Sets are objects but concepts are not objects. We perceive objects and understand concepts. Understanding is a different kind of perception: it is a step in the direction of reduction to the last cause.

14 7.3.4 Trying to see (i.e. understand) a concept more clearly is the correct way to describe the phenomenon vaguely described as "examining what we mean by a word"

15 But, despite their remoteness from sense experience, we do have something like a perception also of the objects of set theory, as is seen from the fact that the axioms force themselves upon us as being true.

\section{Referências bibliográficas}

AZZOUNI, J. 1997. Thick Epistemic Access: Distinguishing the Mathematical from the Empirical. The Journal of Philosophy, v. 94, n.9, pp. 472-484, Sep., 1997.

RODRÍGUEZ-CONSUEGRA, F. A. 1995. Kurt Gödel: unpublished philosophical essays; with a historic-philosophical introduction. Berlin: Birkhäuser Verlag. 
FEFERMAN, S., DAWSON, J. W., KLEENE, S. C., MOORE, G. H., SOLOVAY, R. M. e van HEIJENOORT, J. (Ed.). 1990. Kurt Gödel: Collected works, volume II - Publications 1938 - 1974. New York: Oxford University Press.

FEFERMAN, S., DAWSON, J. W., GOLDFARB, W., PARSONS, C. e SOLOVAY, R. M. (Ed.) 1995. Kurt Gödel: Collected Works, volume III Unpublished Essays and Lectures. New York: Oxford University Press.

GÖDEL, K. *1951. Some Basic theorems on the foundations of mathematics and their implications. In: FEFERMAN, S., DAWSON, J. W., GOLDFARB, W., PARSONS, C. e SOLOVAY, R. M. (Ed.) 1995. Kurt Gödel: Collected Works, volume III - Unpublished Essays and Lectures. New York: Oxford University Press, pp. 304-326.

.1953/9. Is mathematics Syntax of Language?,Versões II e VI. In: RODRÍGUEZ-CONSUEGRA, F. A. 1995. Kurt Gödel: unpublished philosophical essays; with a historic-philosophical introduction. Berlin: Birkhäuser Verlag, pp. 171 - 218.

*1953/9. Is mathematics Syntax of Language?, Versões III e V. In: FEFERMAN, S., DAWSON, J. W., GOLDFARB, W., PARSONS, C. e SOLOVAY, R. M. (Ed.) 1995. Kurt Gödel: Collected Works, volume III - Unpublished Essays and Lectures. New York: Oxford University Press, pp. 334-362.

$\star 1961 /$ ? The Modern Development of the foundations of Mathematics in the Light of Philosophy. In: FEFERMAN, S., DAWSON, J. W., GOLDFARB, W., PARSONS, C. e SOLOVAY, R. M. (Ed.) 1995. Kurt Gödel: Collected Works, volume III - Unpublished Essays and Lectures. New York: Oxford University Press, pp. 374 - 387. 1964. What is Cantor's continuum problem? In: FEFERMAN, S., DAWSON, J. W., KLEENE, S. C., MOORE, G. H., SOLOVAY, R. M. e van HEIJENOORT, J. (Ed.), 1990. Kurt Gödel: Collected works, volume II - Publications 1938 - 1974. New York: Oxford University Press, pp. $254-270$. 
150

PARSONS, C. Platonism and mathematical intuition in Kurt Gödel's thought. The Bulletin of Symbolic Logic, v. 1, n. 1, pp. 44-74, Mar., 1995. WANG, H. 1974. From Mathematics to Philosophy. New York: Humanities Press. 1996. A Logical Journey: From Gödel to Philosophy. Cambridge, Mass: MIT Press. 